\title{
A Sufficient Degree Condition for a Graph to Contain All Trees of Size $k$
}

\author{
Camino BALBUENA \\ Departament de Matemàtica Aplicada III, Universitat Politècnica de Catalunya, \\ C/ Jordi Girona 1-3 (Edifici C2, Despatx 302), 08034, Barcelona, Spain \\ E-mail:m.camino.balbuena@upc.edu
}

\section{Alberto MÁRQUEZ José Ramón PORTILLO}

Departamento de Matemática Aplicada I, Universidad de Sevilla, E.T.S. de Informática, Avda. Reina Mercedes, s/n, 41012, Sevilla, Spain

E-mail:almar@us.es_josera@us.es

\begin{abstract}
The Erdős-Sós conjecture says that a graph $G$ on $n$ vertices and number of edges $e(G)>n(k-$ 1) $/ 2$ contains all trees of size $k$. In this paper we prove a sufficient condition for a graph to contain every tree of size $k$ formulated in terms of the minimum edge degree $\xi(G)$ of a graph $G$ defined as $\xi(G)=$ $\min \{d(u)+d(v)-2: \quad u v \in E(G)\}$. More precisely, we show that a connected graph $G$ with maximum degree $\Delta(G) \geq k$ and minimum edge degree $\xi(G) \geq 2 k-4$ contains every tree of $k$ edges if $d_{G}(x)+d_{G}(y)$ $\geq 2 k-4$ for all pairs $x, y$ of nonadjacent neighbors of a vertex $u$ of $d_{G}(u) \geq k$.
\end{abstract}

Keywords Erdős-Sós conjecture

MR(2000) Subject Classification 05C05, 05C07, 05C35

\section{Introduction}

Erdös and Gallai [1] proved that if the size of graph $G$ on $n$ vertices is at least $e(G)>n(k-1) / 2$, then $G$ contains a path of size $k$. This fact leads Erdős and Sós to formulate the following conjecture.

Conjecture 1 (Erdős-Sós [2]) If $G$ is a graph on n vertices and the number of edges of $G$ is e $(G)$ $>n(k-1) / 2$, then $G$ contains all trees of size $k$.

The Erdös-Sós conjecture is clearly true for stars of size $k$, because if $e(G)>n(k-1) / 2$, then some vertex in $G$ must have degree at least $k$. Furthermore, the conjecture is true for the trees collected in the following theorem.

Theorem A Let $G$ be a graph of order $n$ and size $e(G)>n(k-1) / 2$, where $k \geq 1$ is an integer. Then $G$ contains the following trees of size $k$ :

(i) Paths [1].

Supported by the Ministry of Education and Science, Spain, and the European Regional Development Fund (ERDF) under Projects MTM2008-06620-C03-02 and MTM2008-05866-C03-01, and by the Catalonian Government under Project 1298 SGR2009 and Andalusian Government under Project P06-FQM-01649 
(ii) Comets (trees obtained from a star and a path by identifying one leaf of the star with one leaf of the path) [3].

(iii) Caterpillars. It is mentioned in [4] that this fact was proved by Perles in 1990.

(iv) Trees with a vertex adjacent to $t$ leaves, where $t \geq(k-1) / 2[5]$.

(v) Trees with a vertex adjacent to $t$ leaves, where $t \geq(k-3) / 2[6]$.

(vi) Spiders (trees with a unique vertex of degree greater than two) with three legs or spiders with legs of length at most three [7].

(vii) Trees with diameter four [8].

(viii) Trees with size $k \leq 7[5,6]$.

The items (i)-(iv) were mentioned by Woźniak in [3]. Moreover, the Erdős-Sós conjecture has been proved for both certain values of $k$ and certain graphs gathered in the theorem below.

Theorem B Let $G$ be a graph of order $n$ and size $e(G)>n(k-1) / 2$, where $k \geq 1$ is an integer. Then $G$ contains all trees of size $k$ if one of the following assertions holds:

(i) $k=n-1[9,10] ; k=n-2[11] ; k=n-3$ [3]; $k=n-4$ [12].

(ii) The girth of $G$ is at least 5 [13].

(iii) The graph $G$ does not contain the cycle $C_{4}[14]$.

(iv) The graphs whose complements contain no $C_{4}$ [15].

(v) The graph $G$ does not contain $K_{2,\lfloor k / 18\rfloor}$ as a subgraph $[16,17]$.

(vi) The girth of the complement graph of $G$ is at least 5 [18].

Other recent variations on the Erdős-Sós conjecture can be seen in [19].

\subsection{Our Result}

It is also known that if the minimum degree of $G$ is $\delta(G) \geq k$, then $G$ contains all trees of size $k$ (see Chartrand and Lesniak [20, Theorem 3.8, p. 60]). Clearly if the requirement on the number of edges of Erdős-Sós conjecture is true, then the maximum degree is $\Delta(G) \geq k$. In this note we state that if the minimum degree of a graph $G$ is $\delta(G) \geq k-1$ and the maximum degree is $\Delta(G) \geq k$, then $G$ contains all trees of size $k$.

This is derived as a direct consequence of the following stronger result, formulated in terms of the minimum edge degree $\xi(G)$ of a graph $G$, defined as $\xi(G)=\min \{d(u)+d(v)-2: u v \in E(G)\}$.

Theorem 1.1 Let $k \geq 4$ be an integer and let $G$ be a connected graph with maximum degree $\Delta(G) \geq k$ and minimum edge degree $\xi(G) \geq 2 k-4$. Then $G$ contains every tree of $k$ edges if $d_{G}(x)+d_{G}(y) \geq 2 k-4$ for all pair $x, y$ of nonadjacent neighbors of a vertex $u$ of $d_{G}(u) \geq k$.

As a consequence of Theorem 1.1 the following result is now apparent.

Corollary 1.2 Any graph $G$ with a connected component of minimum degree $\delta(G) \geq k-1$ and maximum degree $\Delta(G) \geq k$ contains every tree of size $k$.

Observe that any pair of adjacent vertices $x, y$ of $K_{k, k-2}$ satisfies $d_{G}(x)+d_{G}(y) \geq k+k-2=$ $2 k-2$ and any pair of vertices of the same partite set of cardinality $k$ are neighbors of a vertex of degree $k$ and this pair satisfies $d_{G}(x)+d_{G}(y) \geq 2 k-4$. Hence the conditions of Theorem 1.1 
hold and so the following result holds.

Corollary 1.3 Let $k \geq 4$ be an integer. Any graph $G$ containing a complete bipartite $K_{k, k-2}$ as a subgraph contains every tree of size $k$.

Theorem 1.1 is in a certain sense stronger than that established by Erdős-Sós conjecture as shown by Corollary 1.3. Indeed, $K_{k, k-2}$ is a graph having fewer edges than required by ErdösSós hypothesis, but applying Theorem 1.1 we obtain that any graph $G$ containing a complete bipartite $K_{k, k-2}$ as a subgraph contains every tree of size $k$.

In the following section we provide the proof of Theorem 1.1.

\section{Proof}

In the proof of Theorem 1.1 we follow the terminology introduced in $[13,14]$ which we recall next. A vertex of degree 1 is a leaf, and a penultimate vertex in a tree is a leaf in the subtree of $T$ obtained by deleting all leaves of $T$. In other words, all the neighbors except one of a penultimate vertex are leaves.

Proof of Theorem 1.1 Note that a star of 4 vertices satisfies the conditions of the theorem for $k=3$, but obviously this graph does not contain a path of length 3 . This is the reason for assuming $k \geq 4$. The proof is by induction on $k$. First let us see that the result is true for $k=4$. Notice that $G$ contains a star of size 4 , since $\Delta(G) \geq k=4$; say $s \in V(G)$ adjacent to $s_{1}, s_{2}, s_{3}, s_{4} \in V(G)$. If the induced subgraph $G\left[s_{1}, s_{2}, s_{3}, s_{4}\right]$ of $G$ has at least two edges, clearly $G$ contains all trees of size 4 . Then we may assume that $G\left[s_{1}, s_{2}, s_{3}, s_{4}\right]$ has at most one edge. Since all pairs $x, y$ of nonadjacent neighbors of a vertex of degree at least 4 satisfy $d_{G}(x)+d_{G}(y) \geq 4$, it follows that $G$ contains a spider with at least two legs of length 2 . This implies that $G$ contains every tree of size $k=4$. Therefore, from now on suppose that $k \geq 5$ and the result is true for all integers less than $k$. Let $T$ be a tree with $k$ edges and $G$ a graph satisfying the hypothesis of the theorem.

Let us choose a penultimate vertex $u$ of $T$ adjacent (in $T$ ) to $r$ leaves with $r$ as small as possible. Denote by $u^{-}$the unique non-leaf vertex of $T$ such that $u^{-} u \in E(T)$. Let $T^{\prime}$ be the tree obtained from $T$ by removing the $r$ leaf-neighbors of vertex $u$, and $T^{\prime \prime}$ the tree obtained from $T^{\prime}$ by removing vertex $u$. Thus, by induction, both trees $T^{\prime}$ and $T^{\prime \prime}$ are subgraphs of $G$. Observe also that $\left|V\left(T^{\prime \prime}\right)\right|=k-r$. We denote $U=N_{G}\left(u^{-}\right) \backslash V\left(T^{\prime \prime}\right)$, clearly $u \in U$. Suppose that there exists some vertex $u^{\prime} \in U$ having at least $r$ neighbors in $G$ outside of $T^{\prime \prime}$. Then by replacing in $T^{\prime}$ vertex $u$ with this vertex $u^{\prime}$ we would obtain a tree subgraph of $G$ isomorphic to $T$ and the result is true. Therefore every vertex $u^{\prime} \in U$ has at most $r-1$ neighbors in $G$ outside of $T^{\prime \prime}$. It follows that $d_{G}\left(u^{\prime}\right) \leq r-1+\left|V\left(T^{\prime \prime}\right)\right|=k-1$ for all $u^{\prime} \in U$; so each $u^{\prime}$ misses (i.e., is not adjacent to) at most one vertex in $T^{\prime \prime}$. Let us distinguish the following cases.

Case $|U| \leq r \quad$ For all $u^{\prime} \in U$ we have

$$
\begin{aligned}
\xi(G) & \leq d_{G}\left(u^{\prime}\right)+d_{G}\left(u^{-}\right)-2 \leq k-1+\left|V\left(T^{\prime \prime}\right)\right|-1+|U|-2 \\
& =2 k+|U|-r-4 \leq 2 k-4 .
\end{aligned}
$$


Since by hypothesis $\xi(G) \geq 2 k-4$ it follows that all the above inequalities are equalities, that is, $\xi(G)=2 k-4,|U|=r, d_{G}\left(u^{\prime}\right)=k-1$ for all $u^{\prime} \in U$ and $d_{G}\left(u^{-}\right)=\left|V\left(T^{\prime \prime}\right)\right|-1+|U|=k-1$. Therefore $\left(V\left(T^{\prime \prime}\right)-\left\{u^{-}\right\}\right) \cup U=N_{G}\left(u^{-}\right)$and also $V\left(T^{\prime \prime}\right) \subseteq N_{G}\left(u^{\prime}\right)$ for all $u^{\prime} \in U$, thus each vertex from $U \cup\left\{u^{-}\right\}$is adjacent in $G$ to all the vertices of $T^{\prime \prime}$.

Observe that $\left|V\left(T^{\prime \prime}\right) \cup U\right|=k$, so the maximum degree of the induced subgraph $G\left[V\left(T^{\prime \prime}\right)\right.$ $\cup U]$ of $G$ is $k-1$. Since by hypothesis $G$ is connected and has maximum degree at least $k$, it follows that $|V(G)| \geq 1+k$ so that some vertex of $G\left[V\left(T^{\prime \prime}\right) \cup U\right]$ must be adjacent to some external vertex.

If some $u_{0}$ of $U$ has $y \in N_{G}\left(u_{0}\right) \backslash\left(V\left(T^{\prime \prime}\right) \cup U\right)$, then we obtain a tree subgraph of $G$ isomorphic to $T$ by changing in $T^{\prime \prime}$ a penultimate vertex $w$ for $u_{0}$ and one of its leaves $w_{i}$ for $y$ (we can do that because $u_{0}$ is adjacent in $G$ to every vertex of $T^{\prime \prime}$ ); by joining vertex $u^{-}$of $T^{\prime \prime}$ with $w$ by an edge (because $u^{-} w \in E(G)$ ) and by joining $w$ with $w_{i}$ and the $r-1$ vertices of $U-u_{0}$, because every vertex of $U-u_{0}$ is adjacent in $G$ with $w \in V\left(T^{\prime \prime}\right)$.

If $N_{G}(U) \subset V\left(T^{\prime \prime}\right) \cup U$ then some $t$ of $T^{\prime \prime}-u^{-}$has $z \in N_{G}(t) \backslash\left(V\left(T^{\prime \prime}\right) \cup U\right)$. We obtain a tree subgraph of $G$ isomorphic to $T$ by changing in $T^{\prime}$ vertex $t$ for $u$, by joining vertex $u^{-}$with vertex $t$ by an edge because $u^{-} t \in E(G)$, and by joining $t$ with $z$ and with $r-1$ vertices of $U$ different from $u$.

In any case, $G$ contains every tree of size $k$ and the theorem holds.

Case $|U| \geq r+1 \quad$ Suppose that there exists some $u_{0} \in U$ with $d_{G}\left(u_{0}\right)=k-1$, then $V\left(T^{\prime \prime}\right) \subseteq N_{G}\left(u_{0}\right)$. Thus by replacing in $T^{\prime \prime}$ vertex $u^{-}$with this vertex $u_{0}$ and by replacing in $T^{\prime}$ vertex $u$ with $u^{-}$, we obtain a tree subgraph of $G$ isomorphic to $T$ because $u^{-}$is adjacent to at least $\left|U-u_{0}\right| \geq r$ vertices outside of $T^{\prime \prime}$. Hence, we may assume that $d_{G}\left(u^{\prime}\right) \leq k-2$ for all $u^{\prime} \in U$. Moreover, since $\xi(G) \geq 2 k-4$ it follows that $d_{G}\left(u^{-}\right) \geq k$ because for all $u^{\prime} \in U$, $\xi(G) \leq d_{G}\left(u^{\prime}\right)+d_{G}\left(u^{-}\right)-2$. Moreover, the vertices of $U$ are independent because otherwise we would have $\xi(G) \leq d_{G}\left(u^{\prime}\right)+d_{G}\left(u^{\prime \prime}\right)-2 \leq 2(k-2)-2$, which is a contradiction. Therefore by the hypothesis of the theorem we have for all $u^{\prime}, u^{\prime \prime} \in U$ that $d_{G}\left(u^{\prime}\right)+d_{G}\left(u^{\prime \prime}\right) \geq 2 k-4$, implying that $d_{G}\left(u^{\prime}\right)=k-2$ for all $u^{\prime} \in U$.

Let us denote $L_{1}=\left\{t \in V\left(T^{\prime \prime}\right): u^{-} t \in E\left(T^{\prime \prime}\right)\right\} \subseteq N_{G}\left(u^{-}\right)$. Note that if $L_{1} \subseteq N_{G}\left(u^{\prime}\right)$ for some $u^{\prime} \in U$, then we obtain again a tree subgraph of $G$ isomorphic to $T$ by replacing vertex $u^{-}$with $u^{\prime}$ in $T^{\prime \prime}$ and $u$ with $u^{-}$in $T^{\prime}$, so assume this is not the case. Then

$$
\text { for each } u^{\prime} \in U \text {, there exists } t_{u^{\prime}} \in L_{1} \text { such that } V\left(T^{\prime \prime}-t_{u^{\prime}}\right) \subseteq N_{G}\left(u^{\prime}\right) \text {. }
$$

Suppose that there is some penultimate $w$ of $T^{\prime \prime}$ such that every vertex of $U$ is adjacent to $w$ and all its leaves, $w_{1}, \ldots, w_{r^{\prime}}$ with $r^{\prime} \geq r$. Let us consider the tree $H$ obtained from $T^{\prime}$ by replacing $w_{1}, \ldots, w_{r}$ with $r$ vertices of $U$ different from $u$. Thus we obtain a tree subgraph of $G$ isomorphic to $T$ by joining vertex $u$ with $w_{1}, \ldots, w_{r}$. Consequently, by (2.1) it only remains to study the case in which every penultimate vertex $w$ of $T^{\prime \prime}$ satisfies $w \in L_{1}$ and $w=t_{u^{\prime}}$ for some $u^{\prime} \in U$.

Let $w \in L_{1}$ be a penultimate vertex in $T$. If there exists some $u_{0} \in U$ such that $w u_{0} \in E(G)$, 
then $d_{G}(w) \geq k$ because $d_{G}\left(u_{0}\right)=k-2$ and $\xi(G) \geq 2 k-4$. In this case let us consider the tree $H$ obtained by interchanging in $T^{\prime}$ vertex $u_{0}$ for $w$, because $u_{0}$ is adjacent to all the leaves of $w$ by (2.1). Since $d_{G}(w) \geq k$, then there are at least $r$ neighbors of $w$ outside of this tree $H$, and hence $G$ contains a tree subgraph isomorphic to $T$. Therefore we may assume that $w$ is independent of every vertex $u^{\prime} \in U$, which implies that $t_{u^{\prime}}=w$ for all $u^{\prime} \in U$, and moreover $w$ is the unique penultimate in $L_{1}$. In this case the other vertex of $L_{1}$, if any, must be a leaf adjacent to every vertex of $U$. Suppose there is a leaf $\ell \in L_{1}$. Then $d_{G}(\ell) \geq k$ (because $u \ell \in E(G)$, $d_{G}(u)=k-2$ and $\left.\xi(G) \geq 2 k-4\right)$ and we obtain a tree subgraph isomorphic to $T$ by changing in $T^{\prime}$ vertex $u$ for $\ell$ and $\ell$ for $u$. Consequently we may suppose that $L_{1}=\{w\}$, yielding that tree $T$ consists of a path $u, u^{-}, w$ of length 2 with $u$ adjacent to $r$ leaves and $w$ adjacent to $k-r-2$ leaves.

Let us consider the tree $R$ formed by $u_{1}, \ldots, u_{r} \in U$ neighbors of $u^{-}$and the path $u^{-}, w, w_{1}$, where $w_{1}$ is a leaf of $w$. We have $d_{G}\left(w_{1}\right) \geq k$ because $\xi(G) \geq 2 k-4$ and by (2.1), $w_{1}$ is adjacent to any vertex $u^{\prime} \in U$ of $d_{G}\left(u^{\prime}\right)=k-2$. Therefore $w_{1}$ must be adjacent to at least $k-r-2$ vertices outside of $R$. Hence the tree $R$ along with these external neighbors of $w_{1}$ is a tree subgraph isomorphic to $T$, and the theorem holds.

Acknowledgements We would like to thank the anonymous referees for their suggestions and for their comments.

\section{References}

[1] Erdős, P., Gallai, T.: On maximal paths and circuits of graphs. Acta Math. Acad. Sci. Hungar., 10, 337-356 (1959)

[2] Erdős, P.: Extremal problems in graph theory. In: Theory of Graphs and its Applications (M. Fiedler Ed.), Academic Press, 1965, 29-36

[3] Woźniak, M.: On the Erdős-Sós conjecture. J. Graph Theory, 21(2), 229-234 (1996)

[4] Moser, W., Pach, J.: Recent developments in combinatorial geometry. In: New Trends in Discrete and Computational Geometry, Springer, New York, 1993

[5] Sidorenko, A. F.: Assymptotic solution for a new class of forbidden r-graphs. Combinatorica, 9(2), 207-215 (1989)

[6] Eaton, N., Tiner, G.: On the Erdős-Sós conjecture and graphs with large minimum degree. Ars Comb., 95, 373-382 (2010)

[7] Fan, G., Sun, L.: The Erdős-Sós conjecture for spiders. Discrete Math., 307, 3055-3062 (2007)

[8] McLennan, A.: The Erdős-Sós conjecture for trees of diameter four. J. Graph Theory, 291-301 (2005)

[9] Sauer, N., Spencer, J.: Edge disjoint placement of graphs. J. Combin. Theory Ser. B, 25, 295-302 (1978)

[10] Bing, Z.: A note on the Erdős-Sós conjecture. Acta Math. Scientia, 4(3), 287-289 (1984)

[11] Slater, P. J., Teo, S. K., Yap, H. P.: Packing a tree with a graph of the same size. J. Graph Theory, 9, 213-216 (1985)

[12] Tiner, G.: On the Erdős-Sós conjecture for graphs on $n=k+3$ vertices. Ars Comb., 95, 143-150 (2010)

[13] Brandt, S., Dobson, E.: The Erdős-Sós conjecture for graphs of girth 5. Discrete Math., 150, 411-414 (1996)

[14] Saclé, J. F., Woźniak, M.: The Erdős-Sós conjecture for graphs without $C_{4}$. J. Combin. Theory Ser. B, 70, 367-372 (1997)

[15] Yin, J. H., Li, J. S.: The Erdős-Sós conjecture for graphs whose complements contain no $C_{4}$. Acta Math. Appl. Sin. Eng. Ser., 20, 397-400 (2004)

[16] Haxell, P. E.: Tree embeddings. J. Graph Theory, 36, 121-130 (2001) 
[17] Dobson, E.: Constructing trees in graphs whose complement has no $K_{2, s}$. Combinatorics, Probability and Computing, 11, 343-347 (2002)

[18] Li, G., Liu, A., Wang, M.: A result of Erdős-Sós conjecture. Ars Comb., 55, 123-127 (2000)

[19] Yin, J. H., Li, J. S.: A variation of a conjecture due to Erdős and Sós. Acta Mathematica Sinica, English Series, 25(5), 795-802 (2009)

[20] Chartrand, G., Lesniak, L.: Graphs and Digraphs, 4th ed., Chapman and Hall, London, 2005 